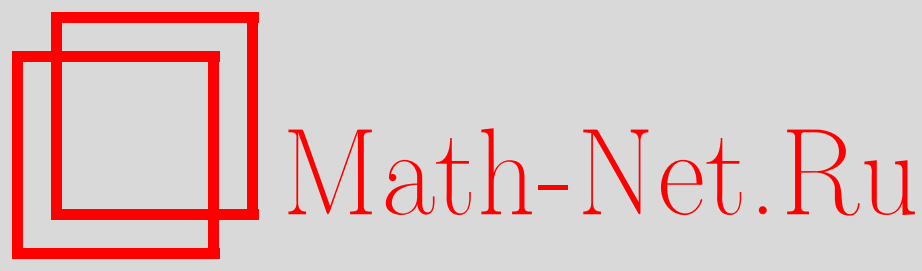

А. Х. Хачатрян, Х. А. Хачатрян, Качественное различие решений для стационарных модельных уравнений Больцмана в линейном и нелинейном случаях, $Т M \Phi$, 2014, том 180, номер 2, 272-288

DOI: https://doi.org/10.4213/tmf8623

Использование Общероссийского математического портала Math-Net.Ru подразумевает, что вы прочитали и согласны с пользовательским соглашением http: //www . mathnet.ru/rus/agreement

Параметры загрузки:

IP : 54.84 .234 .179

26 апреля 2023 г., 08:38:37

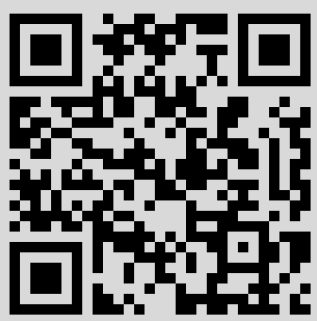




\title{
ФИЗИКА
}

Том 180, № 2

август, 2014

(C) 2014 г.

А. Х. Хачатрян*, Х. А. Хачатрян*

\section{КАЧЕСТВЕННОЕ РАЗЛИЧИЕ РЕШЕНИЙ ДЛЯ СТАЦИОНАРНЫХ МОДЕЛЬНЫХ УРАВНЕНИЙ БОЛЬЦМАНА В ЛИНЕЙНОМ И НЕЛИНЕЙНОМ СЛУЧАЯХ}

\begin{abstract}
Рассматриваются задачи для нелинейного уравнения Больцмана в рамках двух моделей: новой нелинейной модели и модели Батнагара-Гросса-Крука. Соответствующие преобразования сводят эти задачи к нелинейным системам интегральных уравнений. В рамках новой нелинейной модели доказано существование положительного и ограниченного решения нелинейной системы интегральных уравнений. Приведены примеры функций, описывающих нелинейность в случае этой модели. Полученная форма уравнения Больцмана в рамках модели Батнагара-Гросса-Крука позволяет проанализировать задачу и указать способ ее решения. Показано, что существует качественное различие между решениями в линейном и нелинейном случаях: в нелинейном случае температура является ограниченной функцией, в то время как в линейном приближении она линейно возрастает на бесконечности. Установлено, что в рамках новой нелинейной модели уравнения, описывающие распределения температуры, концентрации и среднемассовой скорости, взаимно согласованны, чего нельзя утверждать в случае модели Батнагара-Гросса-Крука.
\end{abstract}

Ключевые слова: модельные уравнения Больцмана, нелинейность, монотонность, ограниченное решение, температурный скачок, система нелинейных интегральных уравнений.

DOI: $10.4231 / \operatorname{tmf} 8623$

Посвящается памяти академика В.С. Владимирова

\section{1. ВВЕДЕНИЕ}

В настоящее время нелинейное кинетическое уравнение Больцмана стало предметом исследования в различных областях физической кинетики, в частности в кинетической теории газов. Одними из центральных задач кинетической теории газов являются классическая задача о течении газа в полупространстве, ограниченном

* Институт математики НАН Республики Армения, Ереван, Армения. E-mail: aghavard@hotbox.ru, khach82@rambler.ru 
твердой стенкой, и связанная с ней задача о распределении температуры, концентрации и среднемассовой скорости газа вблизи стенки. Из-за сложного вида интеграла столкновения в уравнении Больцмана он заменяется различными моделями [1]-[4]. Так, например, проблема температурного скачка в основном рассматривалась в рамках модели Батнагара-Гросса-Крука (БГК) уравнения Больцмана. В этой модели интеграл столкновения заменяется на $f_{0}^{\text {loc }}-f$, где $f$ - искомая функция распределения, a $f_{0}^{\text {loc }}$ - локально-максвелловская функция распределения. Применение модели БГК обычно сопровождается приближенной линеаризацией нелинейного интегродифференциального уравнения. Эта линеаризация априори может существенно исказить задачу не только в количественном, но и в качественном отношении, т. е. привести к асимптотически иному решению, которое отличается от решения исходного нелинейного интегродифференциального уравнения. Тем не менее изучению указанной задачи в линейном приближении посвящены многочисленные работы (см., например, статьи [1], [2], [5]-[15] и ссылки в них).

В работе [16] рассматривается нелинейное уравнение Больцмана в рамках модели БГК для задач течения газа в полупространстве (задача Крамерса). В этой работе показано, что задача точно линеаризуется, и ее решение сводится к решению линейного интегрального уравнения и некоторому простому нелинейному соотношению. В работе [17] рассматривается задача Крамерса в рамках одной нелинейной модели и доказывается существование положительного и ограниченного решения. Показано, что учет нелинейной зависимости интеграла столкновения от функции распределения приводит к асимптотически иному решению первоначального уравнения.

В настоящей работе мы рассматриваем задачу о течении газа в полупространстве, ограниченном твердой стенкой, в рамках двух нелинейных моделей: в рамках новой нелинейной (НH) модели, предложенной нами, и модели БГК, причем последняя рассматривается как в нелинейном, так и в линейном случаях. В рамках ННмодели доказано существование положительного и ограниченного решения. Приведены примеры функций, описывающих нелинейность в случае НН-модели. В рамках модели БГК показано, что имеется качественное различие между решениями в линейном и нелинейном случаях: в нелинейном случае температура есть ограниченная функция, в то время как в линейном случае она линейно растет на бесконечности. Установлено, что в НН-модели нелинейные уравнения, описывающие распределения температуры, концентрации и среднемассовой скорости, взаимно согласованны, а в в случае модели БГК этого утверждать нельзя.

\section{2. СВЕДЕНИЕ УРАВНЕНИЯ БОЛЬЦМАНА К НЕЛИНЕЙНОЙ СИСТЕМЕ ИНТЕГРАЛЬНЫХ УРАВНЕНИЙ}

Пусть полупространство $x>0$, заполненное газом, ограничено твердой плоской стенкой: плоскостью $x=0$. Введем декартову систему координат. Предполагается что газ течет со среднемассовой скоростью $\vec{U}(x)=(0, U(x), 0)$ по положительному направлению оси $O y$. Обозначим через $f(x, \vec{s})$ искомую функцию распределения частиц по скоростям $\vec{s}=\left(s_{1}, s_{2}, s_{3}\right)$. В силу предполагаемой плоской симметрии задачи функция распределения не зависит от $y$ и $z$. 
Стационарное уравнение Больцмана в рамках предложенной нелинейной модели записывается в виде

$$
s_{1} \frac{\partial f(x, \vec{s})}{\partial x}+f(x, \vec{s})=R\left(f_{1}(x), f_{2}(x), U(x), \vec{s}\right),
$$

где функции $f_{1}(x), f_{2}(x)$ и $U(x)$ выражаются через моменты функции распределения:

$$
\begin{aligned}
f_{1}(x) & =\iint_{-\infty}^{\infty} \int_{-\infty} f(x, \vec{s}) d^{3} s=n(x), \\
f_{2}(x) & =\frac{2}{3} \iint_{-\infty}^{\infty}(\vec{s}-\vec{U})^{2} f(x, \vec{s}) d^{3} s \equiv n(x) T(x), \\
U(x) f_{1}(x) & =\iint_{-\infty}^{\infty} \int_{2} f(x, \vec{s}) d^{3} s .
\end{aligned}
$$

Здесь $n(x)$ и $T(x)$ - концентрация и температура газа соответственно, $x$ - расстояние от стенки.

В дальнейшем будем рассматривать следующие две модели:

1) НН-модель

$$
R\left(f_{1}(x), f_{2}(x), U(x), \vec{s}\right) \sim \frac{1}{\pi^{3 / 2}} e^{-(\vec{s}-\vec{U}(x))^{2}} G\left(f_{1}(x), f_{2}(x), U(x)\right),
$$

где функция $G\left(f_{1}, f_{2}, u\right)$ удовлетворяет определенным условиям (см. теорему 1$)$;

2) модель БГК (см., например, работу [18])

$$
R\left(f_{1}(x), f_{2}(x), U(x), \vec{s}\right)=\frac{f_{1}^{5 / 2}(x) e^{-(\vec{s}-\vec{U}(x))^{2} f_{1}(x) / f_{2}(x)}}{\left(\pi f_{2}(x)\right)^{3 / 2}}=\frac{n(x)}{(\pi T(x))^{3 / 2}} e^{-(\vec{s}-\vec{U}(x))^{2} / T(x)} .
$$

K уравнению (1) добавим граничные условия на стенке. Ниже мы уточним условие, которому должна удовлетворять функция распределения на ограничивающей твердой стенке.

Введем функции $f^{-}(x, \vec{s})$ и $f^{+}(x, \vec{s})$, описывающие распределения по скоростям молекул, двигающихся соответственно в сторону стенки и от нее:

$$
\begin{aligned}
& f^{-}(x, \vec{s})= \begin{cases}0, & \text { если } s_{1}<0, \\
f\left(x,-s_{1}, s_{2}, s_{3}\right), & \text { если } s_{1} \geqslant 0 .\end{cases} \\
& f^{+}(x, \vec{s})= \begin{cases}f(x, \vec{s}), & \text { если } s_{1} \geqslant 0, \\
0, & \text { если } s_{1}<0,\end{cases}
\end{aligned}
$$

Присутствие стенки нарушает равновесие газа. Молекулы газа взаимодействуют между собой, а некоторая часть из них взаимодействует со стенкой, что и обуславливает нелинейную зависимость интеграла столкновения от функции распределения частиц. Предположим, что частицы не претерпевают на стенке каких-либо химических превращений. Пусть молекула, падающая на стенку со скоростью $\vec{p}$, 
отражается от нее с некоторой скоростью $\vec{s}$. Обозначим через $K(\vec{s}, \vec{p}) d^{3} s d^{3} p$ вероятность того, что молекула, падая на поверхность стенки со скоростью $\vec{p}$, лежащей в объеме $d^{3} p$, отлетит от нее со скоростью $\vec{s}$, лежащей в объеме $d^{3} s$. Очевидно, что

$$
\int_{-\infty}^{\infty} \int_{-\infty}^{\infty} \int_{0}^{\infty} K(\vec{s}, \vec{p}) d s_{3} d s_{2} d s_{1}=1
$$

Мы имеем

$$
f^{+}(0, \vec{s})=\int_{-\infty}^{\infty} \int_{-\infty}^{\infty} \int_{0}^{\infty} K(\vec{s}, \vec{p}) \frac{p_{1}}{s_{1}} f^{-}\left(0,-p_{1}, p_{2}, p_{3}\right) d p_{3} d p_{2} d p_{1}
$$

Одним из наиболее распространенных способов построения приближений функции распределения отраженных молекул является предположение, что часть молекул, доля которых от полного числа молекул составляет $1-\varepsilon$, отражается от стенки зеркально, остальные (доля которых равна $\varepsilon$ ) поглощаются, а потом диффузно отражаются, имея при этом распределение Максвелла по скоростям. Здесь $\varepsilon$ называется коэффициентом аккомодации, $0<\varepsilon \leqslant 1$.

Предположим, что температура отраженных молекул равна заданной температуре стенки, которую примем равной единице. При этом имеем

$$
K(\vec{s}, \vec{p})=(1-\varepsilon) \delta(\vec{s}-\vec{p}) \frac{s_{1}}{p_{1}}+\frac{2 \varepsilon e^{-s^{2}} s_{1}}{\pi},
$$

где $\delta(x)$ - функция Дирака. Тогда из соотношений $(6),(7)$ получим

$$
f^{+}(0, \vec{s})=(1-\varepsilon) f^{-}(0, \vec{s})+\frac{2 \varepsilon e^{-s^{2}}}{\pi} \int_{-\infty}^{\infty} \int_{-\infty}^{\infty} \int_{0}^{\infty} p_{1} f^{-}\left(0, p_{1}, p_{2}, p_{3}\right) d p_{3} d p_{2} d p_{1} .
$$

Отметим, что в этом равенстве учтено условие непротекания частиц (число падающих молекул должно быть равным числу отраженных молекул).

Для простоты в дальнейшем мы будем использовать граничное условие (8). Подчеркнем, что результаты, полученные в настоящей работе, можно обобщить на случай общего закона отражения (6). В качестве второго граничного условия зададим поведение функции распределения $f^{-}(x, \vec{s})$ на бесконечности по $x$ :

$$
f^{-}(x, \vec{s})=o\left(e^{x / s_{1}}\right), \quad x \rightarrow \infty .
$$

С учетом формул (3)-(5), (8), (9) из уравнения (1) получаем

$$
\begin{aligned}
& f^{+}(x, \vec{s})=C^{+}(\vec{s}) e^{-x / s_{1}}+\int_{0}^{x} e^{-(x-t) / s_{1}} \psi(t, \vec{s}) \frac{d t}{s_{1}} \\
& f^{-}(x, \vec{s})=\int_{x}^{\infty} e^{(t-x) / s_{1}} \psi(t, \vec{s}) \frac{d t}{s_{1}}
\end{aligned}
$$


где

$$
\begin{aligned}
C^{+}(\vec{s})= & (1-\varepsilon) \int_{0}^{\infty} e^{-t / s_{1}} \psi(t, \vec{s}) \frac{d t}{s_{1}}+ \\
& +\frac{2 \varepsilon e^{-s^{2}}}{\pi} \int_{-\infty}^{\infty} \int_{-\infty}^{\infty} \int_{0}^{\infty}\left[\int_{0}^{\infty} e^{-t / p_{1}} \psi(t, \vec{p}) d t\right] d p_{3} d p_{2} d p_{1},
\end{aligned}
$$

функция $\psi(t, \vec{s})$ в случае НН-модели имеет вид

$$
\psi(t, \vec{s})=\frac{1}{\pi^{3 / 2}} e^{-(\vec{s}-\vec{U}(t))^{2}} G\left(f_{1}(t), f_{2}(t), U(t)\right)
$$

а в случае модели БГК

$$
\psi(t, \vec{s})=\frac{1}{\pi^{3 / 2}} \frac{e^{-(\vec{s}-\vec{U}(t))^{2} f_{1}(t) / f_{2}(t)} f_{1}^{5 / 2}(t)}{f_{2}^{3 / 2}(t)} .
$$

2.1. НН-модель. Подставляя соотношения (10)- (12) в формулы (2), интегрируя по $s_{2}$ и $s_{3}$ от $-\infty$ до $\infty$, с помощью элементарных, но трудоемких преобразований приходим к следующей системе нелинейных интегральных уравнений относительно функций $f_{1}(x), f_{2}(x)$ и $U(x)$ :

$$
\begin{gathered}
f_{i}(x)=C_{\mathrm{HH}}\left(f_{1} ; f_{2}\right) g_{i}(x)+ \\
+\int_{0}^{\infty}\left(K_{i}(x-t)+(1-\varepsilon) K_{i}(x+t)\right) G\left(f_{1}(t), f_{2}(t), U(t)\right) d t, \quad i=1,2, \\
U(x) f_{1}(x)=\int_{0}^{\infty}\left(K_{1}(x-t)+(1-\varepsilon) K_{1}(x+t)\right) U(t) G\left(f_{1}(t) ; f_{2}(t), U(t)\right) d t .
\end{gathered}
$$

Здесь

$$
\begin{gathered}
C_{\mathrm{HH}}\left(f_{1}, f_{2}\right)=\int_{0}^{\infty} K_{0}(x) G\left(f_{1}(x) ; f_{2}(x) ; U(x)\right) d x \\
g_{1}(x)=\frac{\varepsilon}{\sqrt{\pi}} \int_{0}^{\infty} e^{x / s} e^{-s^{2}} d s, \quad g_{2}(x)=\frac{2 \varepsilon}{3 \sqrt{\pi}} \int_{0}^{\infty} e^{-x / s} e^{-s^{2}}\left(s^{2}+1\right) d s, \\
K_{0}(x)=2 \int_{0}^{\infty} e^{-x / s} e^{-s^{2}} d s, \quad x \geqslant 0 \\
K_{1}(x)=\frac{1}{\sqrt{\pi}} \int_{0}^{\infty} e^{-|x| / s} e^{-s^{2}} \frac{d s}{s}, \\
K_{2}(x)=\frac{2}{3 \sqrt{\pi}} \int_{0}^{\infty} e^{-|x| / s} e^{-s^{2}}\left(s^{2}+1\right) \frac{d s}{s} .
\end{gathered}
$$

Функции $K_{i}(x), i=1,2$, удовлетворяют условию консервативности: они положительны и

$$
\int_{-\infty}^{\infty} K_{i}(x) d x=1
$$


2.2. Нелинейная модель БГК. Подставляя соотношения (10) в формулы (2) с учетом равенств (13), (11), получим следующую нелинейную систему интегральных уравнений типа Урысона:

$$
\begin{aligned}
f_{i}(x) & =C_{\text {БГК }}\left(f_{1} ; f_{2}\right) g_{i}(x)+\int_{0}^{\infty} W_{i}\left(x, t, f_{1}(t), f_{2}(t)\right) d t, \quad i=1,2, \\
U(x) f_{1}(x) & =\int_{0}^{\infty} W_{1}\left(x, t, f_{1}(t), f_{2}(t)\right) U(t) d t .
\end{aligned}
$$

Здесь

$$
\begin{aligned}
C_{\text {БГК }}\left(f_{1} ; f_{2}\right) & =2 \iint_{0}^{\infty} \frac{f_{1}^{3 / 2}(t)}{\sqrt{f_{2}(t)}} e^{-t / 1} e^{-s_{1}^{2} f_{1}(t) / 2(t)} d t d s_{1}= \\
& =2 \iint_{0}^{\infty} \frac{n(t)}{\sqrt{T(t)}} e^{-t / s_{1}} e^{-s_{1}^{2} / T(t)} d s_{1} d t
\end{aligned}
$$

и

$$
\begin{aligned}
& W_{1}\left(x, t, f_{1}(t), f_{2}(t)\right)=\frac{f_{1}^{3 / 2}(t)}{\sqrt{\pi f_{2}(t)}} \times \\
& \times \int_{0}^{\infty}\left(e^{-|x-t| / s_{1}}+(1-\varepsilon) e^{-(x+t) / s_{1}}\right) e^{-s_{1}^{2} f_{1}(t) / f_{2}(t)} \frac{d s_{1}}{s_{1}} \\
& W_{2}\left(x, t, f_{1}(t), f_{2}(t)\right)=\frac{2}{3 \sqrt{\pi}} \sqrt{f_{1}(t) f_{2}(t)} \times \\
& \quad \times \int_{0}^{\infty}\left(t\left[e^{-|x-t| / s_{1}}+(1-\varepsilon) e^{-(x+t) / s_{1}}\right) e^{-s_{1}^{2} f_{1}(t) / f_{2}(t)}\left(\frac{s_{1}^{2} f_{1}(t)}{f_{2}(t)}+1\right) \frac{d s_{1}}{s_{1}} .\right.
\end{aligned}
$$

Перепишем $W_{1}$ и $W_{2}$ в терминах концентрации и температуры. Имеем

$$
\begin{aligned}
& W_{1}\left(x, t, f_{1}(t), f_{2}(t)\right)=n(t) \widetilde{W}_{1}(x, t, T(t)), \\
& W_{2}\left(x, t, f_{1}(t), f_{2}(t)\right)=n(t) T(t) \widetilde{W}_{2}(x, t, T(t)),
\end{aligned}
$$

где

$$
\begin{aligned}
\widetilde{W}_{1}(x, t, T(t))= & \frac{1}{\sqrt{\pi T(t)}} \int_{0}^{\infty}\left(e^{-|x-t| / s_{1}}+(1-\varepsilon) e^{-(x+t) / s_{1}}\right) e^{-s_{1}^{2} / T(t)} \frac{d s_{1}}{s_{1}}, \\
\widetilde{W}_{2}(x, t, T(t))= & \frac{2}{3 \sqrt{\pi T(t)}} \times \\
& \times \int_{0}^{\infty}\left(e^{-|x-t| / s_{1}}+(1-\varepsilon) e^{-(x+t) / s_{1}}\right) e^{-s_{1}^{2} / T(t)}\left(\frac{s_{1}^{2}}{T(t)}+1\right) \frac{d s_{1}}{s_{1}} .
\end{aligned}
$$




\section{3. НН-МОДЕЛЬ. ОБ ОДНОМ ПОДХОДЕ К РЕШЕНИЮ НЕЛИНЕЙНОЙ СИСТЕМЫ}

Уравнения (14) и (15) слабо связаны. При любом заданном виде функции $U(x)$, фигурирующей в выражении для $G\left(f_{1}(t), f_{2}(t), U(t)\right)$, система (14) отделяется от уравнения (15). Положим $G\left(f_{1}(x), f_{2}(x), U(x)\right) \equiv G\left(f_{1}(x), f_{2}(x), x\right)$. При любых заданных $f_{1}(x)$ и $f_{2}(x)$, фигурирующих в выражении для $G\left(f_{1}(x), f_{2}(x), U(x)\right)$, уравнение (15) преобразуется к следующему скалярному нелинейному интегральному уравнению относительно $f_{3}(x)=U(x) f_{1}(x)$ :

$$
f_{3}(x)=\int_{0}^{\infty}\left(K_{1}(x-t)+(1-\varepsilon) K_{1}(x+t)\right) G_{1}\left(f_{3}(t), t\right) d t .
$$

Здесь введено следующее обозначение:

$$
G_{1}\left(f_{3}(x), x\right) \equiv \frac{f_{3}(x)}{f_{1}(x)} G\left(f_{1}(x), f_{2}(x), \frac{f_{3}(x)}{f_{1}(x)}\right),
$$

где $f_{1}(x)$ и $f_{2}(x)$ - наперед заданные функции.

Сначала рассмотрим систему (14) при заданной функции $U(x)$. Справедлива

Теорема 1. Пусть функиия $G\left(f_{1}, f_{2}, x\right)$, заданная на множестве $\mathbb{R} \times \mathbb{R} \times \mathbb{R}^{+}$, принимает вещественные значения и удовлетворяет условию критичности:

$$
G(0,0, x)=0, \quad x \in \mathbb{R}^{+} .
$$

Предположим, что существуют числа $\eta_{i} \geqslant 1 / 2, i=1,2$, такие, что:

1) $G\left(\eta_{1}, \eta_{2}, x\right) \leqslant \min \left(\eta_{1}, \eta_{2}\right)$ для всех $x \in \mathbb{R}^{+}$,

2) $G\left(g_{1}(x), g_{2}(x), x\right) \geqslant 1$ для всех $x \in \mathbb{R}^{+}$,

3) при каждом фиксированном $x \in \mathbb{R}^{+}$функиия $G\left(f_{1}, f_{2}, x\right)$ монотонно возрасmaem nо $f_{1}$ u $f_{2}$, когда $f_{1} \in\left[g_{1}(x), \eta_{1}\right]$ u $f_{2} \in\left[g_{2}(x), \eta_{2}\right]$,

4) $G\left(f_{1}, f_{2}, x\right)$ удовлетворлет условию Каратеодори по совокупности аргументов $\left(f_{1}, f_{2}\right)$ на множестве $\left[0, \eta_{1}\right] \times\left[0, \eta_{2}\right] \times \mathbb{R}^{+}$, т.е. при каждом фиксированном $\left(f_{1}, f_{2}\right) \in\left[0, \eta_{1}\right] \times\left[0, \eta_{2}\right]$ функиия $G\left(f_{1}, f_{2}, x\right)$ измерима по $x$ на $\mathbb{R}^{+}$и при почти всех $x \in \mathbb{R}^{+}$функиия $G\left(f_{1}, f_{2}, x\right)$ непрерывна по совокупности аргументов $\left(f_{1}, f_{2}\right)$ на множестве $\left[0, \eta_{1}\right] \times\left[0, \eta_{2}\right]$.

Тогда нелинейная система (14), кроме тривиального решения, имеет положительное и ограниченное решение.

ДоКАЗАТЕЛЬСтво. Введем следующие специальные итерации: пусть для $i=1,2$

$$
\begin{aligned}
& \quad f_{i}^{(0)}(x)=g_{i}(x), \quad x \geqslant 0, \\
& f_{i}^{(n+1)}(x)=C_{\mathrm{HH}}\left(f_{1}^{(n)} ; f_{2}^{(n)}\right) g_{i}(x)+ \\
& +\int_{0}^{\infty}\left(K_{i}(x-t)+(1-\varepsilon) K_{i}(x+t)\right) G\left(f_{1}^{(n)}(t) ; f_{2}^{(n)}(t), t\right) d t, \quad n=0,1,2, \ldots .
\end{aligned}
$$

С помощью индукции по $n$ докажем, что последовательность $\left\{f_{i}^{(n)}(x)\right\}_{n=0}^{\infty}$ монотонно возрастает по $n$ для каждого $i=1,2$. Сначала убедимся, что

$$
f_{i}^{(1)}(x) \geqslant f_{i}^{(0)}(x), \quad x \in \mathbb{R}^{+} .
$$


С учетом формулы (16) и условий 2, 3 теоремы из соотношений (28) имеем

$$
\begin{aligned}
f_{i}^{(1)}(x)= & g_{i}(x) \int_{0}^{\infty} K_{0}(y) G\left(f_{1}^{(0)}(y) ; f_{2}^{(0)}(y), y\right) d y+ \\
& +\int_{0}^{\infty}\left(K_{i}(x-t)+(1-\varepsilon) K_{i}(x+t)\right) G\left(f_{1}^{(0)}(t) ; f_{2}^{(0)}(t), t\right) d t \geqslant \\
\geqslant & g_{i}(x) \int_{0}^{\infty} K_{0}(y) G\left(g_{1}(y), g_{2}(y), y\right) d y \geqslant g_{i}(x) \int_{0}^{\infty} K_{0}(y) d y=g_{i}(x),
\end{aligned}
$$

ибо $\int_{0}^{\infty} K_{0}(y) d y=1$ (см. формулу $\left.(18)\right)$ и

$$
f_{i}^{(0)}(x)=g_{i}(x) \leqslant \frac{1}{2} \leqslant \eta_{i}
$$

Теперь докажем, что

$$
f_{i}^{(1)}(x) \leqslant \eta_{i}, \quad i=1,2
$$

Действительно, с учетом условий 1, 3 теоремы и неравенств (29) из соотношений (28) получим

$$
\begin{aligned}
f_{i}^{(1)}(x) \leqslant & g_{i}(x) \int_{0}^{\infty} K_{0}(y) G\left(\eta_{1}, \eta_{2}, y\right) d y+ \\
& +\int_{0}^{\infty}\left(K_{i}(x-t)+(1-\varepsilon) K_{i}(x+t)\right) G\left(\eta_{1}, \eta_{2}, t\right) d t \leqslant \\
\leqslant & \min \left(\eta_{1}, \eta_{2}\right)\left(g_{i}(x) \int_{0}^{\infty} K_{0}(y) d y+\int_{0}^{\infty}\left(K_{i}(x-t)+(1-\varepsilon) K_{i}(x+t)\right) d t\right)= \\
= & \min \left(\eta_{1}, \eta_{2}\right)\left(g_{i}(x)+1-\int_{x}^{\infty} K_{i}(t) d t+(1-\varepsilon) \int_{x}^{\infty} K_{i}(t) d t\right) \leqslant \\
\leqslant & \eta_{i}\left(g_{i}(x)+1-\varepsilon \int_{x}^{\infty} K_{i}(t) d t\right)=\eta_{i}
\end{aligned}
$$

так как

$$
\int_{0}^{\infty} K_{0}(y) d y=1, \quad g_{i}(x)=\varepsilon \int_{x}^{\infty} K_{i}(t) d t, \quad i=1,2 .
$$

Пусть $f_{i}^{(n)}(x) \geqslant f_{i}^{(n-1)}(x)$ при некотором натуральном $n \in \mathbb{N}$ и $f_{i}^{(n)}(x) \leqslant \eta_{i}$, $i=1,2$, для всех $x \in \mathbb{R}^{+}$. Тогда в силу монотонности функции $G\left(f_{1}, f_{2}, x\right)$ по $\left(f_{1}, f_{2}\right)$ с учетом условия 1 теоремы из (28) получаем

$$
\begin{aligned}
f_{i}^{(n+1)}(x) & \geqslant C\left(f_{1}^{(n-1)} ; f_{2}^{(n-1)}\right) g_{i}(x)+ \\
& +\int_{0}^{\infty}\left(K_{i}(x-t)+(1-\varepsilon) K_{i}(x+t)\right) G\left(f_{1}^{(n-1)}(t), f_{2}^{(n-1)}(t), t\right) d t=f_{i}^{(n)}(x)
\end{aligned}
$$

Оценка $f_{i}^{(n+1)}(x) \leqslant \eta_{i}$ получается аналогично неравенству (30). 
Следовательно, функциональная последовательность $\left\{f_{i}^{(n)}(x)\right\}_{n=0}^{\infty}$ имеет поточечный предел:

$$
\lim _{n \rightarrow \infty} f_{i}^{(n)}(x)=f_{i}(x), \quad i=1,2,
$$

причем предельные функции $f_{1}(x)$ и $f_{2}(x)$ удовлетворяют системе $(14)$ в силу условия 4 теоремы и предельной теоремы Леви.

Из условия монотонного возрастания последовательности $\left\{f_{i}^{(n)}(x)\right\}_{n=0}^{\infty}$ по $n$, учитывая неравенства $f_{i}^{(n)}(x) \leqslant \eta_{i}$, справедливые для всех $n=0,1,2, \ldots$ и всех $x \in \mathbb{R}^{+}$, получаем

$$
g_{i}(x) \leqslant f_{i}(x) \leqslant \eta_{i}, \quad i=1,2, \quad x \geqslant 0 .
$$

Теорема доказана.

ПримеР 1. Приведем пример функции $G\left(f_{1}, f_{2}, x\right)$. Пусть

$$
G\left(f_{1}(x), f_{2}(x), x\right)=\frac{G_{0}\left(f_{1}(x), f_{2}(x)\right)}{G_{0}\left(f_{1}(x), f_{2}(x)\right)+\alpha(x)} \frac{G_{0}\left(g_{1}(x), g_{2}(x)\right)+\alpha(x)}{G_{0}\left(g_{1}(x), g_{2}(x)\right)},
$$

где $G_{0}\left(f_{1}(x), f_{2}(x)\right)=\delta \cdot \widetilde{G}_{0}\left(f_{1}(x), f_{2}(x)\right) \min \left(\eta_{1}, \eta_{2}\right), \delta \in(0,1)$, а $\alpha(x)$ - измеримая функция, удовлетворяющая двойному неравенству

$$
0<\alpha(x) \leqslant\left(\min \left(\eta_{1}, \eta_{2}\right)-1\right) G_{0}\left(g_{1}(x), g_{2}(x)\right), \quad \eta_{i}>1, \quad i=1,2 ;
$$

функция $\widetilde{G}_{0}\left(f_{1}(x), f_{2}(x)\right)$ может быть задана любым из следующих равенств:

$$
\begin{aligned}
& \widetilde{G}_{0}\left(f_{1}(x), f_{2}(x)\right)=\frac{f_{1}^{p}(x)}{\eta_{1}^{p}} \frac{f_{2}^{q}(x)}{\eta_{2}^{q}}, \quad p, q \geqslant 1, \\
& \widetilde{G}_{0}\left(f_{1}(x), f_{2}(x)\right)=\frac{\ln \left(f_{1}(x) f_{2}(x)+1\right)}{\ln \left(\eta_{1} \eta_{2}+1\right)}, \\
& \widetilde{G}_{0}\left(f_{1}(x), f_{2}(x)\right)=\frac{f_{1}(x) f_{2}(x) e^{f_{1}(x) f_{2}(x)}}{\eta_{1} \eta_{2} e^{\eta_{1} \eta_{2}}}, \\
& \widetilde{G}_{0}\left(f_{1}(x), f_{2}(x)\right)=\frac{f_{1}(x) f_{2}(x)}{f_{1}(x)+f_{2}(x)+1} \frac{\eta_{1}+\eta_{2}+1}{\eta_{1} \eta_{2}} .
\end{aligned}
$$

Теперь рассмотрим уравнение (27). Справедлива

ТЕОрема 2. Пусть существуют положительные числа $\eta>0$ и $0<\xi<\eta / 2$ такие, что:

1) $G_{1}(t, \eta) \leqslant \eta, G(t, \xi) \geqslant 2 \xi$ npu $t \in \mathbb{R}^{+}$,

2) $G_{1}\left(t, f_{3}\right)$ монотонно возрастает по $f_{3}$ на отрезке $[\xi, \eta]$,

3) $G_{1}\left(t, f_{3}\right)$ удовлетворяет условию Каратеодори по $f_{3}$ на множестве $\mathbb{R}^{+} \times[\xi, \eta]$.

Тогда уравнение (27) с ядром вида (19) имеет положительное решение из пространства существенно ограниченных функций на $\mathbb{R}^{+}$. 
ДокАЗАТЕЛьство аналогично доказательству теоремы 1 из работы [19].

ПримеР 2. Приведем пример функции $G_{1}\left(t, f_{3}\right)$ :

$$
G_{1}\left(t, f_{3}\right)=\rho(t) \sqrt{f_{3}}, \quad \rho \in C[0, \infty), \quad 2 \sqrt{\xi} \leqslant \rho(t) \leqslant \sqrt{\eta}, \quad \eta \geqslant 4 \xi>0 .
$$

В качестве $\eta$ и $\xi$ можно выбрать

$$
\eta=\sup _{t \geqslant 0} \rho^{2}(t), \quad \xi=\underset{t \geqslant 0}{\operatorname{essinf}} \frac{\rho^{2}(t)}{4} .
$$

Рассмотрим частный случай, когда функция $G_{1}\left(f_{3}, x\right)$ явно не зависит от $x$, т. е. $G_{1}\left(f_{3}, x\right) \equiv G_{2}(U)$, это соответствует предположению, что концентрация и температура вблизи у стенки постоянны.

Имеет место следующее утверждение [19].

УтВеРЖДЕНИЕ 1. Предположим, что функиия $G_{2}(z)$ удовлетворяет следующим условиям: существуют числа $\eta$ и $\xi, \eta>2 \xi$, такие, что $G_{2}(\eta)=\eta, G_{2}(\xi)=2 \xi$, функиия $G_{2}(z)$ монотонно возрастает и удовлетворяет условию Каратеодории на на отрезке $[\xi, \eta]$, где $\eta$ - первый положительный корень уравнения $G_{2}(z)=z$. Тогда уравнение

$$
U(x)=\int_{0}^{\infty}\left(K_{1}(x-t)+(1-\varepsilon) K_{1}(x+t)\right) G_{2}(U(t)) d t
$$

имеет положительное и ограниченное решение. Более того, это решение монотонно возрастает, удовлетворяет двойному неравенству $\xi \leqslant U(x) \leqslant \eta$, и его предел при $x \rightarrow \infty$ конечен, причем

$$
\lim _{x \rightarrow \infty} U(x)=\eta
$$

В качестве $G(z)$ можно выбрать следующие функции:

$$
\begin{array}{llll}
G_{2}(z)=z^{1 / p}, & z>0, & \eta=1, & \xi=\left(\frac{1}{2}\right)^{p /(p-1)}, \quad p>1, \\
G_{2}(z)=a e^{-(z-a)^{2}}, & z>0, & & \xi \in\left(0, \frac{\eta}{2}\right), \\
G_{2}(z)=e^{z-1}, & \eta=1, &
\end{array}
$$

в последнем случае $\xi$ - положительный корень уравнения $e^{z-1}=2 z$. Число $\eta$ имеет важный физический смысл: оно представляет собой коэффициент изотермического скольжения [1], [2], [4], [6].

\section{4. МОДЕЛЬ БГК. ЛИНЕЙНОЕ ПРИБЛИЖЕНИЕ}

Применение модели БГК обычно сопровождается приближенной линеаризацией системы нелинейных уравнений (21)-(25). В линейном приближении концентрация $n(x)$ и температура $T(x)$ представимы в виде

$$
\Delta n(x)=1+\Delta f_{1}(x), \quad \Delta T(x)=1+\Delta f_{2}(x),
$$


где $\Delta f_{i}(x), i=1,2,-$ поправки к функции концентрации и температуры соответственно.

Заменяя в соотношениях (23), (26) соответствующие функции суммой первых двух членов разложения в степенной ряд по $n(x)$ и $T(x)$, сведем исходную нелинейную систему $(21),(22)$ к скалярному интегральному уравнению относительно среднемассовой скорости $U(x)$,

$$
U(x)=\int_{0}^{\infty}\left(K_{1}(x-t)+(1-\varepsilon) K_{1}(x+t)\right) U(t) d t
$$

и к системе линейных интегральных уравнений с симметричным и знакопеременным $(2 \times 2)$-матричным ядром относительно $\Delta f_{1}(x)$ и $\Delta f_{2}(x)$,

$$
\Delta f_{i}(x)=\widetilde{C} g_{i}(x)+\sum_{j=1}^{2} \int_{0}^{\infty}\left(K_{i j}(x-t)+(1-\varepsilon) K_{i j}(x+t)\right) \Delta f_{j}(t) d t, \quad i=1,2
$$

где

$$
\begin{gathered}
K_{i j}(x)=\frac{1}{\sqrt{\pi}} \int_{0}^{\infty} e^{-|x| / s} G_{i j}(s) d s \\
\widetilde{C}=2 \sum_{i=1}^{2} \int_{0}^{\infty} \tilde{g}_{i}(t) \Delta f_{i}(t) d t \\
\tilde{g}_{1}(x)=\int_{0}^{\infty} e^{-x / s} e^{-s^{2}} d s, \quad \tilde{g}_{2}(x)=\int_{0}^{\infty} e^{-x / s} e^{-s^{2}}\left(s^{2}-\frac{1}{2}\right) d s, \\
G_{11}(s)=\frac{1}{\sqrt{\pi} s} e^{-s^{2}}, \quad G_{22}(s)=\frac{2}{3 \sqrt{\pi} s} e^{-s^{2}}\left[\left(s^{2}-\frac{1}{2}\right)^{2}+1\right] \\
G_{21}(s)=G_{12}(s)=\frac{1}{\sqrt{\pi} s} e^{-s^{2}}\left(s^{2}-\frac{1}{2}\right) .
\end{gathered}
$$

Знакопеременность матричного ядра обусловлена отсутствием монотонности исходного нелинейного интегрального оператора (подробнее см. в разделе 5 ).

Изучению и точному решению консервативного линейного уравнения (32) посвящена работа [16]. В этой работе доказано, что уравнение (32), кроме тривиального решения, обладает положительным решением с асимптотикой $O(x)$ при $x \rightarrow \infty$.

Из равенства (34) следует, что матричное ядро $K(x)=\left(K_{i j}(x)\right)_{i, j=1,2}$ удовлетворяет условию

$$
\int_{-\infty}^{\infty} K(x) d x=I
$$

где $I$ - единичная матрица.

Пусть $\bar{K}(s)$ - преобразование Фурье от $K(x)$ :

$$
\bar{K}(s)=\int_{-\infty}^{\infty} K(x) e^{i s x} d x, \quad s \in \mathbb{R}
$$

(преобразование Фурье понимается покомпонентно). Из формул (34), (37), (38) следует, что $\operatorname{det}|I-\bar{K}(s)|=0$. В работах [7], [12], [13] показано, что символ оператоpa $I-\bar{K}(s)$ имеет ноль четвертого порядка в точке $s=0$. Последнее означает, что 
соответствующий интегральный оператор необратим в достаточно широких функциональных пространствах.

Из-за такой высокой степени вырожденности система (33) выпадает из общей теории систем интегральных уравнений Винера-Хопфа-Ганкеля и относится к особым случаям таких систем. Существуют многочисленные работы, посвященные изучению задачи температурного скачка в линейном приближении (см., например, работы [6], [7], [12], [13], [15] и ссылки в них). В статьях [7], [13] для системы интегральных уравнений (33) с необратимым интегральным оператором показано, что удается выделить необратимые факторы из исходного оператора и свести задачу к новой системе со сжимающим интегральным оператором. В работе [12] предложена аналитическая версия метода дискретных ординат для решения задачи о температурном скачке в рамках линеаризованной модели БГК уравнения Больцмана. Во всех указанных выше работах получено положительное решение с асимптотикой $O(x)$ при $x \rightarrow \infty$.

На самом деле в силу линейности легко можно убедиться, что решение системы (33) можно представить в виде

$$
\Delta f_{i}(x)=\widetilde{C} \psi_{i}(x)+\varphi_{i}(x), \quad i=1,2,
$$

где $\psi_{i}(x)$ - решение линейной системы неоднородных уравнений

$$
\psi_{i}(x)=g_{i}(x)+\sum_{j=1}^{2} \int_{0}^{\infty}\left(K_{i j}(x-t)+(1-\varepsilon) K_{i j}(x+t)\right) \psi_{j}(t) d t, \quad i=1,2,
$$

а $\varphi_{i}(x), i=1,2,-$ решение соответствующей системы однородных уравнений

$$
\varphi_{i}(x)=\sum_{j=1}^{2} \int_{0}^{\infty}\left(K_{i j}(x-t)+(1-\varepsilon) K_{i j}(x+t)\right) \varphi_{j}(t) d t, \quad i=1,2 .
$$

С учетом формул (36) из системы (40) получаем

$$
\widetilde{C}=\frac{B}{1-A}, \quad \text { где } \quad A \neq 1
$$

здесь

$$
B=2 \sum_{j=1}^{2} \int_{0}^{\infty} \varphi_{j}(t) \tilde{g}_{j}(t) d t, \quad A=2 \sum_{j=1}^{2} \int_{0}^{\infty} \tilde{g}_{j}(t) \psi_{j}(t) d t
$$

Равенство (42) можно получить непосредственно из формулы (39) с учетом (35).

Известно [7], что неоднородная система (40) имеет положительное ограниченное решение, а однородная система (41) имеет нетривиальное положительное решение с асимптотикой $O(x)$ при $x \rightarrow \infty$. Если мы выберем тривиальное решение $\varphi_{i}=0$, однородного уравнения $(41)$, то $\widetilde{C}=0$, т. е. мы приходим к тривиальному решению системы (33). Из представления (39) следует, что $\Delta f_{i}(x)=1+\Delta f_{i}(x)=O(x)$ при $x \rightarrow \infty$. Таким образом, линеаризация лишает нас возможности получить ограниченное решение. Линейный рост температуры на бесконечности не может адекватно описать задачу с физической точки зрения. Негативная тенденция повышения температуры вдали от стенки является следствием линеаризации модели БГК уравнения Больцмана. 


\section{5. НЕЛИНЕЙНАЯ МОДЕЛЬ БГК. ОБ ОДНОМ ПОДХОДЕ К РЕШЕНИЮ НЕЛИНЕЙНОЙ СИСТЕМЫ}

Наша ближайшая цель - построение положительного ограниченного решения системы (21), (22). Исходная задача достаточно сложна. С одной стороны, мы имеем нелинейную систему интегральных уравнений с некомпактными операторами, с другой стороны, эти операторы не обладают свойством монотонности, что весьма усложняет проблему построения положительных неподвижных точек для этих операторов. Тем не менее полученная форма уравнений Больцмана $(21),(22)$ позволяет проанализировать задачу и указать эффективный способ ее решения. Функции $\widetilde{W}_{1}$ и $\widetilde{W}_{2}$, задаваемые равенствами (26), обладают замечательными свойствами: они положительны (в отличие от линейного случая) и удовлетворяют соотношениям

$$
\int_{0}^{\infty} \widetilde{W}_{i}(x, t, T(t)) d x \leqslant 1, \quad \sup _{t \geqslant 0} \int_{0}^{\infty} \widetilde{W}_{i}(x, t, T(t)) d x=1, \quad i=1,2,
$$

при всех $T(t)$.

Заметим, что при любом заданном виде функции $f_{1}(x)$, фигурирующей в выражении (25) для $W_{2}$ уравнение (21) при $i=2$ представляет собой скалярное нелинейное интегральное уравнение типа Урысона относительно функции $f_{2}(x)$, а при любом заданном виде функции $f_{2} / f_{1}$, фигурирующей в выражении (24) для $W_{1}$ уравнения (21) при $i=1$ и (22) представляют собой разделенные линейные интегральные уравнения относительно функций $f_{1}(x)$ и $f_{3}(x)=U(x) f_{1}(x)$. Заметим также,что не только нулевой $\left(f_{1}, f_{2}\right)^{\mathrm{T}}=(0 ; 0)^{\mathrm{T}}$ вектор-столбец, но и единичный $\left(f_{1}, f_{2}\right)^{\mathrm{T}}=(1 ; 1)^{\mathrm{T}}$ (здесь и далее $\mathrm{T}$ - знак транспонирования) удовлетворяют системе (21), так как в последнем случае

$$
C_{\text {БГК }}\left(f_{1} ; f_{2}\right) \equiv 1, \quad g_{i}(x)=1-\int_{0}^{\infty} W_{i}(x, t, 1,1) d t, \quad i=1,2 .
$$

Поскольку наша цель - построение ограниченного решения системы (21), предположим, что температура и концентрация остаются ограниченными при $x \rightarrow \infty$, т. е. существуют

$$
\sup _{x \geqslant 0} T(x)=\alpha, \quad \sup _{x \geqslant 0} n(x)=\beta,
$$

где числа $\alpha$ и $\beta$ будем считать заданными. Вышеуказанные факты наводят нас на следующую схему решения системы (21).

1. Определить функционал $C_{\text {Бгк }}\left(f_{1} ; f_{2}\right) \equiv c$, который порождается граничным условием на стенке вида (8) и играет роль свободного параметра. Свободный параметр $c$, который подлежит определению, имеет следующий физический смысл: это число частиц, отраженных от стенки.

2. Заменить функцию $T(x)$, входящую в $\widetilde{W}_{1}$ (см. уравнение $(21)$ ), ее максимальным значением $\alpha$.

Тогда уравнение (21) при $i=1$ преобразуется в консервативное линейное интегральное уравнение Винера-Хопфа-Ганкеля

$$
f_{1}(x)=c g_{1}(x)+\int_{0}^{\infty}\left(\widetilde{W}_{1}(x-t)+(1-\varepsilon) \widetilde{W}_{1}(x+t)\right) f_{1}(t) d t
$$


с ядром

$$
\widetilde{W}_{1}(x)=\frac{1}{\sqrt{\pi \alpha}} \int_{0}^{\infty} e^{-|x| / s} e^{-s^{2} / \alpha} \frac{d s}{s} .
$$

Изучению и решению данного уравнения посвящены работы [5], [20], [21]. В них доказано не только существование положительного и ограниченного решения уравнения (43), но и предложен эффективный метод построения этого решения с использованием нелинейных уравнений факторизации и уравнения Амбарцумяна. Так, в работе [21] доказано существование предела $\beta_{0}$ решения на бесконечности, который выражается через интеграл свободного члена $c g_{1}(x)$ и через второй момент ядра $\widetilde{W}_{1}(x)$, т. е. предел $\beta_{0}$ и параметры $c, \alpha$ взаимосвязаны. Последний факт устанавливает связь между средним числом $c$ отраженных от стенки молекул и значением концентрации $\beta_{0}$ на бесконечности. Фактически мы имеем не что иное, как задачу со свободным граничным условием.

Пусть $f_{1}(x)$ - построенное выше положительное и ограниченное решение уравнения (43). Далее мы построим ограниченное решение уравнения (21) при $i=2$, которое при выше сделанных предположениях принимает вид

$$
f_{2}(x)=c g_{2}(x)+\int_{0}^{\infty} W_{2}\left(x, t, f_{1}(t), f_{2}(t)\right) d t .
$$

Данное уравнение представляет собой скалярное нелинейное интегральное уравнение Урысона относительно искомой функции $f_{2}(x)$.

Имеет место следующая

Теорема 3. Пусть $f_{1}(x)$ - заданная положительная ограниченная функиия, $M=\sup _{x \geqslant 0} f_{1}(x)$ и $c$ - заданное положительное число. Тогда нелинейное интегральное уравнение (44) имеет положительное и ограниченное решение $f_{2}(x)$. Более того, справедливы следующие утверждения:

1) если $0<c<1, M \leqslant 1$, то $\operatorname{cg}_{2}(x) \leqslant f_{2}(x) \leqslant 1$ для всех $x \in \mathbb{R}^{+}$;

2) если $c>1, M \leqslant 1$, mo

$$
c g_{2}(x) \leqslant f_{2}(x) \leqslant\left(\frac{c+\sqrt{c^{2}+4}}{2}\right)^{2}, \quad x \in \mathbb{R}^{+} ;
$$

3) если $c>0, M \geqslant 1$, mo

$$
c g_{2}(x) \leqslant f_{2}(x) \leqslant\left(\frac{c+\sqrt{c^{2}+4 M}}{2}\right)^{2}, \quad x \in \mathbb{R}^{+} .
$$

ДокАзАтельство. Начнем с доказательства утверждения 3 теоремы. Заметим, что

$$
W_{2}\left(x, t, f_{1}(t), f_{2}(t)\right) \leqslant W_{2}\left(x, t, M, f_{2}(t)\right)
$$

в силу монотонности $W_{2}$ по третьему аргументу. Покажем, что $W_{2}\left(x, t, f_{1}(t), f_{2}(t)\right)$ монотонно возрастает по четвертому аргументу, т. е. функция $W_{2}\left(x, t, f_{1}(t), z\right)$ монотонно возрастает по $z$. Рассмотрим функцию

$$
\varphi(z)=\left(\frac{s^{2}}{z}+1\right) \sqrt{z} e^{-s^{2} / z}, \quad z \in \mathbb{R}^{+} .
$$


Мы имеем $\varphi^{\prime}(z)>0$ при $z \in \mathbb{R}^{+}$, следовательно, $\varphi(z)$ монотонно возрастает по $z$ на $[0, \infty)$. Из представления функции $W_{2}$ вытекает, что она монотонно возрастает по $z$.

Рассмотрим следующие итерации для уравнения (44):

$$
\begin{aligned}
f_{2}^{(0)}(x) & =c_{0}=\left(\frac{c+\sqrt{c^{2}+4 M}}{2}\right)^{2}, \\
f_{2}^{(n+1)}(x) & =c g_{2}(x)+\int_{0}^{\infty} W_{2}\left(x, t, f_{1}(t), f_{2}^{(n)}(t)\right) d t, \quad n=0,1,2, \ldots .
\end{aligned}
$$

Сначала докажем, что функция $f_{2}^{(n)}(x)$ монотонно убывает по $n$ и $f_{2}^{(n)}(x) \geqslant c g_{2}(x)$.

Пусть $n=0$. Имеем

$$
\begin{aligned}
f_{2}^{(1)}(x)= & c g_{2}(x)+\int_{0}^{\infty} W_{2}\left(x, t, f_{1}(t), c_{0}\right) d t \leqslant \\
\leqslant & \frac{2 \varepsilon}{3 \sqrt{\pi}} c \int_{0}^{\infty} e^{-x / s} e^{-s^{2}}\left(s^{2}+1\right) d s+c_{0}- \\
& -\frac{2 \varepsilon}{3} \sqrt{\frac{c_{0} M}{\pi}} \int_{0}^{\infty} e^{-s^{2} M / c_{0}}\left(\frac{s^{2} M}{c_{0}}+1\right) e^{-x / s} d s \equiv \mathfrak{I}(x) .
\end{aligned}
$$

Покажем, что $\mathfrak{I}(x) \leqslant c_{0}$ для всех $x \in \mathbb{R}^{+}$. Тогда последнее неравенство будет эквивалентно неравенству

$$
c \int_{0}^{\infty} e^{-s^{2}} e^{-x / s}\left(s^{2}+1\right) d s \leqslant \sqrt{c_{0} M} \int_{0}^{\infty} e^{-x / s}\left(\frac{s^{2} M}{c_{0}}+1\right) e^{-s^{2} M / c_{0}} d s,
$$

для чего достаточно доказать, что при каждом $x \in \mathbb{R}^{+}$имеет место неравенство

$$
c e^{-s^{2}}\left(s^{2}+1\right) \leqslant \sqrt{c_{0} M} e^{-s^{2} M / c_{0}}\left(\frac{s^{2} M+c_{0}}{c_{0}}\right) .
$$

Рассмотрим функцию

$$
\varphi\left(s^{2}\right)=c \sqrt{c_{0}} e^{s^{2} M /\left(c_{0}-1\right)}\left(s^{2}+1\right), \quad s^{2} \in \mathbb{R}^{+} .
$$

Легко можно убедиться, что $s_{0}^{2}=M /\left(c_{0}-M\right)$ является единственной точкой максимума функции $\varphi$. Следовательно,

$$
\begin{aligned}
\varphi\left(s^{2}\right) & \leqslant \varphi\left(s_{0}^{2}\right)=c \sqrt{c_{0}}\left(\frac{M}{c_{0}-M}+1\right) e^{-M / c_{0}} \leqslant \\
& \leqslant c\left(\frac{c+\sqrt{c^{2}+4 M}}{2}\right) c_{0}\left[\left(\frac{c+\sqrt{c^{2}+4 M}}{2}\right)^{2}-M\right]^{-1}= \\
& =c_{0} \leqslant c_{0}+s^{2} \leqslant c_{0}+M s^{2} \leqslant \sqrt{M}\left(c_{0}+M s^{2}\right),
\end{aligned}
$$

ибо $M>1$ и $s \in \mathbb{R}^{+}$. Из оценок (49) и (48) мы получаем неравенство (47).

Итак, $\Im(x) \leqslant c_{0}$. Из равенства $(45)$ следует, что $f_{2}^{(1)}(x) \leqslant f_{2}^{(0)}(x)$. Предположим, что $f_{2}^{(n)}(x) \leqslant f_{2}^{(n-1)}(x)$ для некоторого натурального $n \in \mathbb{N}$. Поскольку $W(x, t, \tau, z)$ монотонно возрастает по четвертому аргументу $z$, из формулы (46) следует, что

$$
f_{2}^{(n+1)}(x) \leqslant c g_{2}(x)+\int_{0}^{\infty} W\left(x, t, f_{1}(t), f_{2}^{(n-1)}(t)\right) d t=f_{2}^{(n)}(x) .
$$


Теперь покажем, что для любого $n=0,1,2, \ldots$ имеет место неравенство

$$
f_{2}^{(n)}(x) \geqslant c g_{2}(x)
$$

Сначала проверим неравенство (50) для $n=0$. Имеем

$$
f_{2}^{(0)}(x)=c_{0}=\left(\frac{c+\sqrt{c^{2}+4 M}}{2}\right)^{2} \geqslant \frac{c}{2} \geqslant c g_{2}(x),
$$

поскольку

$$
g_{2}(x) \leqslant \frac{2 \varepsilon}{3 \sqrt{\pi}} \int_{0}^{\infty} e^{-s^{2}}\left(s^{2}+1\right) d s \leqslant \frac{1}{2} \quad \text { при } \quad 0<\varepsilon \leqslant 1 .
$$

Предполагая, что неравенство (50) имеет место для некоторого $n \in \mathbb{N}$ и используя монотонность функции $W_{2}$ по четвертому аргументу, из (46) получаем

$$
f_{2}^{(n+1)}(x) \geqslant c g_{2}(x)+\int_{0}^{\infty} W_{2}\left(x, t, f_{1}(t), c g_{2}(t)\right) d t \geqslant c g_{2}(x) .
$$

Таким образом, последовательность функций $\left\{f_{2}^{(n)}(x)\right\}_{n=0}^{\infty}$ имеет поточечный предел при $n \rightarrow \infty$, причем предельная функция удовлетворяет двойному неравенству

$$
c g_{2}(x) \leqslant f_{2}(x) \leqslant\left(\frac{c+\sqrt{c^{2}+4 M}}{2}\right)^{2} .
$$

В силу теоремы Леви функция $f_{2}(x)$ является решением уравнения (46). Доказательство утверждений 1 и 2 теоремы проводится аналогично с учетом следующих фактов:

1) если $M \leqslant 1,0<c \leqslant 1$, то в качестве нулевого приближения необходимо выбрать $f_{2}^{(0)}(x)=c_{0} \equiv 1$;

2) если же $c>1, M<1$, то $f_{2}^{(0)}(x)=c_{0} \equiv\left(\left(c+\sqrt{c^{2}+4}\right) / c\right)^{2}$.

ЗАмечаниЕ 1. В случае задачи “без течения" (при $U=0$ ) система (21) вполне согласованна, т. е. уравнения (43), (44), порождаемые системой $(21)$, кроме нулевого $\left(f_{1}, f_{2}\right)^{\mathrm{T}}=(0 ; 0)^{\mathrm{T}}$ и единичного $\left(f_{1}, f_{2}\right)^{\mathrm{T}}=(1 ; 1)^{\mathrm{T}}$ решений, имеют положительное ограниченное решение. Иначе обстоит дело в случае $U>0$, когда для любой наперед заданной функции $f_{2}(x)$ уравнение $(22)$ обладает решением, линейно возрастающим на бесконечности. Это означает, что предположения $\sup _{x \geqslant 0} T(x)<\infty$ и $U>0$ несовместимы. Данное противоречие порождается моделью БГК и остается в тени при линеаризации. Следует отметить, что НН-модель лишена этого недостатка.

ЗАмЕчАниЕ 2. Как уже отмечалось, в рамках линейной модели БГК температура, концентрация и среднемассовая скорость линейно растут на бесконечности. Численные расчеты показывают, что решения у стенки (в окрестности нуля) в линейном и нелинейном случаях близки, а вдали от стенки они асимптотически разные. Вопросам разработки основанного на результатах теорем 1-3 алгоритма численного решения задачи для НН-модели и модели БГК в нелинейном случае предполагается посвятить отдельную работу. 
Благодарности. Авторы выражают благодарность рецензенту за полезные замечания. Работа выполнена при финансовой поддержке Государственного комитета по науке Министерства образования и науки Республики Армения (научный проект № SCS 13-1A068).

\section{Список литературы}

[1] К. Черчиньяни, Теория и приложения уравнения Больимана, Мир, М., 1978.

[2] М.Н. Коган, Динамика разреженного газа, Наука, М., 1967.

[3] P. L. Bhatnagar, E. P. Gross, M. Krook, Phys. Rev., 94:3 (1954), 511-525.

[4] Н. Б. Енгибарян, А. Х. Хачатрян, Матем. моделирование, 16:1 (2004), 67-74.

[5] Н.Б. Енгибарян, А.Х. Хачатрян, Ж. вычисл. матем. и матем. физ., 38:3 (1998), $466-482$.

[6] А.В. Латышев, А.А. Юшканов, Аналитическое решение граничных задач для кинетической теории, МГОУ, М., 2004.

[7] Ц.Э. Терджян, А.Х. Хачатрян, Ж. вычисл. матем. и матем. физ., 49:4 (2009), $715-721$.

[8] А. Х. Хачатрян, С. М. Андриян, Матем. моделирование, 16:2 (2004), 31-42.

[9] M. M. R. Williams, Mathematical Methods in Particle Transport Theory, Butterworth, London, 1971.

[10] И. Н. Ларина, В. А. Рыков, ЖК. вычисл. матем. и матем. физ., 51:11 (2011), 2084-2095.

[11] S. K. Loyallka, Phys. Fluids, 14 (1971), 2291-2294.

[12] L. B. Barichello, C. E. Siewert, European J. Appl. Math., 11:4 (2000), 353-364.

[13] N.B. Yengibaryan, A. Kh. Khachatryan, "On temperature and density jumps in kinetic theory of gases", Horizons in World Physics, 240, ed. A. Reimer, Nova Science Publisher, New York, 2003, 103-117.

[14] L. B. Barichello, A. C. R. Bartz, M. Camargo, C. E. Siewert, Phys. Fluids, 14:1 (2002), 382-391.

[15] С. М. Андриян, А.Х. Хачатрян, Ж. вычисл. матем. и матем. физ., 45:11 (2005), 2061-2069.

[16] Н. Б. Енгибарян, А. Х. Хачатрян, ТМФ, 125:2 (2000), 339-342.

[17] А. Х. Хачатрян, Х. А. Хачатрян, ТMФ, 172:3 (2012), 497-504.

[18] C. Villani, Commun. Math. Phys., 234:3 (2003), 455-490.

[19] A. Kh. Khachatryan, Kh. A. Khachatryan, Mem. Differ. Equ. Math. Phys., 51 (2010), $59-72$.

[20] Л.Г. Арабаджян, Н.Б. Енгибарян, “Уравнения в свертках и нелинейные функциональные уравнения", Итоги науки и техн. Сер. Матем. анал., 22, ВИНИТИ, М., 1984, $175-244$.

[21] А.Х. Хачатрян, Некоторые линейные и нелинейные задачи физической кинетики, Дис. ... докт. физ.-матем. наук, Бюракан. астрофиз. обсерватория НАН Армении, Бюракан, обл. Арагацотн, Армения, 1997.

Поступила в редакцию 9.12.2013, после доработки 16.01.2014 